\title{
A Linear Difference Scheme for Dissipative Symmetric Regularized Long Wave Equations with Damping Term
}

\author{
Jinsong $\mathrm{Hu}^{1}{ }^{1}$ Youcai $\mathrm{Xu}{ }^{2}$ and Bing $\mathrm{Hu}^{2}$ \\ ${ }^{1}$ School of Mathematics and Computer Engineering, Xihua University, Chengdu 610039, China \\ ${ }^{2}$ School of Mathematics, Sichuan University, Chengdu 610064, China
}

Correspondence should be addressed to Youcai Xu, xyc@scu.edu.cn

Received 24 August 2010; Accepted 14 November 2010

Academic Editor: V. Shakhmurov

Copyright (C) 2010 Jinsong Hu et al. This is an open access article distributed under the Creative Commons Attribution License, which permits unrestricted use, distribution, and reproduction in any medium, provided the original work is properly cited.

We study the initial-boundary problem of dissipative symmetric regularized long wave equations with damping term by finite difference method. A linear three-level implicit finite difference scheme is designed. Existence and uniqueness of numerical solutions are derived. It is proved that the finite difference scheme is of second-order convergence and unconditionally stable by the discrete energy method. Numerical simulations verify that the method is accurate and efficient.

\section{Introduction}

A symmetric version of regularized long wave equation (SRLWE),

$$
\begin{gathered}
u_{t}+\rho_{x}+u u_{x}-u_{x x t}=0, \\
\rho_{t}+u_{x}=0,
\end{gathered}
$$

has been proposed to model the propagation of weakly nonlinear ion acoustic and space charge waves [1]. The $\sec ^{2}$ solitary wave solutions are

$$
\begin{aligned}
& u(x, t)=\frac{3\left(v^{2}-1\right)}{v} \sec ^{2} \frac{1}{2} \sqrt{\frac{v^{2}-1}{v^{2}}}(x-v t), \\
& \rho(x, t)=\frac{3\left(v^{2}-1\right)}{v^{2}} \sec ^{2} \frac{1}{2} \sqrt{\frac{v^{2}-1}{v^{2}}}(x-v t) .
\end{aligned}
$$


The four invariants and some numerical results have been obtained in [1], where $v$ is the velocity, $v^{2}>1$. Obviously, eliminating $\rho$ from (1.1), we get a class of SRLWE:

$$
u_{t t}-u_{x x}+\frac{1}{2}\left(u^{2}\right)_{x t}-u_{x x t t}=0
$$

Equation (1.3) is explicitly symmetric in the $x$ and $t$ derivatives and is very similar to the regularized long wave equation that describes shallow water waves and plasma drift waves $[2,3]$. The SRLW equation also arises in many other areas of mathematical physics [4-6]. Numerical investigation indicates that interactions of solitary waves are inelastic [7]; thus, the solitary wave of the SRLWE is not a solution. Research on the wellposedness for its solution and numerical methods has aroused more and more interest. In [8], Guo studied the existence, uniqueness, and regularity of the numerical solutions for the periodic initial value problem of generalized SRLW by the spectral method. In [9], Zheng et al. presented a Fourier pseudospectral method with a restraint operator for the SRLWEs and proved its stability and obtained the optimum error estimates. There are other methods such as pseudospectral method, finite difference method for the initial-boundary value problem of SRLWEs (see [915]).

In applications, the viscous damping effect is inevitable, and it plays the same important role as the dispersive effect. Therefore, it is more significant to study the dissipative symmetric regularized long wave equations with the damping term

$$
\begin{gathered}
u_{t}+\rho_{x}-v u_{x x}+u u_{x}-u_{x x t}=0, \\
\rho_{t}+u_{x}+\gamma \rho=0
\end{gathered}
$$

where $v, \gamma$ are positive constants, $v>0$ is the dissipative coefficient, and $\gamma>0$ is the damping coefficient. Equations (1.4)-(1.5) are a reasonable model to render essential phenomena of nonlinear ion acoustic wave motion when dissipation is considered. Existence, uniqueness, and wellposedness of global solutions to (1.4)-(1.5) are presented (see [16-20]). But it is difficult to find the analytical solution to (1.4)-(1.5), which makes numerical solution important.

To authors' knowledge, the finite difference method to dissipative SRLWEs with damping term (1.4)-(1.5) has not been studied till now. In this paper, we propose linear three level implicit finite difference scheme for (1.4)-(1.5) with

$$
u(x, 0)=u_{0}(x), \quad \rho(x, 0)=\rho_{0}(x), \quad x \in\left[x_{L}, x_{R}\right],
$$

and the boundary conditions

$$
u\left(x_{L}, t\right)=u\left(x_{R}, t\right)=0, \quad \rho\left(x_{L}, t\right)=\rho\left(x_{R}, t\right)=0, \quad t \in[0, T] .
$$

We show that this difference scheme is uniquely solvable, convergent, and stable in both theoretical and numerical senses.

Lemma 1.1. Suppose that $u_{0} \in H^{1}, \rho_{0} \in L_{2}$, the solution of (1.4)-(1.7) satisfies $\|u\|_{L_{2}} \leq C,\left\|u_{x}\right\|_{L_{2}} \leq$ $C,\|\rho\|_{L_{2}} \leq C$, and $\|u\|_{L_{\infty}} \leq C$, where $C$ is a generic positive constant that varies in the context. 
Proof. Let

$$
E(t)=\|u\|_{L_{2}}^{2}+\left\|u_{x}\right\|_{L_{2}}^{2}+\|\rho\|_{L_{2}}^{2}=\int_{x_{L}}^{x_{R}} u^{2} \mathrm{~d} x+\int_{x_{L}}^{x_{R}}\left(u_{x}\right)^{2} \mathrm{~d} x+\int_{x_{L}}^{x_{R}} \rho^{2} \mathrm{~d} x, \quad t \in[0, T] .
$$

Multiplying (1.4) by $u$ and integrating over $\left[x_{L}, x_{R}\right]$, we have

$$
\int_{x_{L}}^{x_{R}}\left(u u_{t}+u \rho_{x}-v u u_{x x}+u^{2} u_{x}-u u_{x x t}\right) \mathrm{d} x=0
$$

According to

$$
\begin{gathered}
\int_{x_{L}}^{x_{R}} u u_{t} \mathrm{~d} x=\frac{1}{2} \frac{d}{d t} \int_{x_{L}}^{x_{R}} u^{2} \mathrm{~d} x, \\
\int_{x_{L}}^{x_{R}} u \rho_{x} \mathrm{~d} x=\left.u \rho\right|_{x_{L}} ^{x_{R}}-\int_{x_{L}}^{x_{R}} \rho \mathrm{d} u=-\int_{x_{L}}^{x_{R}} u_{x} \rho \mathrm{d} x, \\
-\int_{x_{L}}^{x_{R}} u u_{x x} \mathrm{~d} x=-\left.u u_{x}\right|_{x_{L}} ^{x_{R}}+\int_{x_{L}}^{x_{R}} u_{x} \mathrm{~d} u=\int_{x_{L}}^{x_{R}}\left(u_{x}\right)^{2} \mathrm{~d} x, \\
\int_{x_{L}}^{x_{R}} u^{2} u_{x} \mathrm{~d} x=\left.\frac{1}{3} u^{3}\right|_{x_{L}} ^{x_{R}}=0, \\
-\int_{x_{L}}^{x_{R}} u u_{x x t} \mathrm{~d} x=-\left.u u_{x t}\right|_{x_{L}} ^{x_{R}}+\int_{x_{L}}^{x_{R}} u_{x t} \mathrm{~d} u=\frac{1}{2} \frac{d}{d t} \int_{x_{L}}^{x_{R}}\left(u_{x}\right)^{2} \mathrm{~d} x,
\end{gathered}
$$

we get

$$
\frac{d}{d t} \int_{x_{L}}^{x_{R}}\left(u^{2}+u_{x}^{2}\right) \mathrm{d} x-2 \int_{x_{L}}^{x_{R}} u_{x} \rho \mathrm{d} x+2 v \int_{x_{L}}^{x_{R}}\left(u_{x}\right)^{2} \mathrm{~d} x=0
$$

Then, multiplying (1.5) by $\rho$ and integrating over $\left[x_{L}, x_{R}\right]$, we have

$$
\int_{x_{L}}^{x_{R}}\left(\rho \rho_{t}+\rho u_{x}+\gamma \rho^{2}\right) \mathrm{d} x=0
$$

By

$$
\int_{x_{L}}^{x_{R}} \rho \rho_{t} \mathrm{~d} x=\frac{1}{2} \frac{d}{d t} \int_{x_{L}}^{x_{R}} \rho^{2} \mathrm{~d} x
$$

we get

$$
\frac{d}{d t} \int_{x_{L}}^{x_{R}} \rho^{2} \mathrm{~d} x+2 \int_{x_{L}}^{x_{R}} u_{x} \rho \mathrm{d} x+2 \gamma \int_{x_{L}}^{x_{R}} \rho^{2} \mathrm{~d} x=0
$$


Adding (1.14) to (1.11), we obtain

$$
\frac{d}{d t} \int_{x_{L}}^{x_{R}}\left(u^{2}+u_{x}^{2}+\rho^{2}\right) \mathrm{d} x=-2 v \int_{x_{L}}^{x_{R}}\left(u_{x}\right)^{2} \mathrm{~d} x-2 \gamma \int_{x_{L}}^{x_{R}} \rho^{2} \mathrm{~d} x \leq 0
$$

So $E(t)$ is decreasing with respect to $t$, which implies that $E(t)=\|u\|_{L_{2}}^{2}+\left\|u_{x}\right\|_{L_{2}}^{2}+\|\rho\|_{L_{2}}^{2} \leq E(0)$, $t \in[0, T]$. Then, it indicates that $\|u\|_{L_{2}} \leq C,\left\|u_{x}\right\|_{L_{2}} \leq C$, and $\|\rho\|_{L_{2}} \leq C$. It is followed from Sobolev inequality that $\|u\|_{L_{\infty}} \leq C$.

\section{Finite Difference Scheme and Its Error Estimation}

Let $h$ and $\tau$ be the uniform step size in the spatial and temporal direction, respectively. Denote $x_{j}=x_{L}+j h(j=0,1,2, \ldots, J), t_{n}=n \tau(n=0,1,2, \ldots, N), N=[T / \tau], u_{j}^{n} \approx u\left(x_{j}, t_{n}\right), \rho_{j}^{n} \approx$ $\rho\left(x_{j}, t_{n}\right)$, and $Z_{h}^{0}=\left\{u=\left(u_{j}\right) \mid u_{0}=u_{J}=0, j=0,1,2, \ldots, J\right\}$. We define the difference operators as follows:

$$
\begin{gathered}
\left(u_{j}^{n}\right)_{x}=\frac{u_{j+1}^{n}-u_{j}^{n}}{h}, \quad\left(u_{j}^{n}\right)_{\bar{x}}=\frac{u_{j}^{n}-u_{j-1}^{n}}{h}, \quad\left(u_{j}^{n}\right)_{\widehat{x}}=\frac{u_{j+1}^{n}-u_{j-1}^{n}}{2 h}, \quad\left(u_{j}^{n}\right)_{\hat{t}}=\frac{u_{j}^{n+1}-u_{j}^{n-1}}{2 \tau}, \\
\bar{u}_{j}^{n}=\frac{u_{j}^{n+1}+u_{j}^{n-1}}{2}, \quad\left\langle u^{n}, v^{n}\right\rangle=h \sum_{j=0}^{J-1} u_{j}^{n} v_{j}^{n}, \quad\left\|u^{n}\right\|^{2}=\left\langle u^{n}, u^{n}\right\rangle, \quad\left\|u^{n}\right\|_{\infty}=\max _{0 \leq j \leq J-1}\left|u_{j}^{n}\right| .
\end{gathered}
$$

Then, the average three-implicit finite difference scheme for the solution of (1.4)-(1.7) is as follow:

$$
\begin{gathered}
\left(u_{j}^{n}\right)_{\widehat{t}}-\left(u_{j}^{n}\right)_{x \bar{x} \widehat{t}}+\left(\rho_{j}^{n}\right)_{\widehat{x}}-v\left(\bar{u}_{j}^{n}\right)_{x \bar{x}}+\frac{1}{3}\left[u_{j}^{n}\left(\bar{u}_{j}^{n}\right)_{\widehat{x}}+\left(u_{j}^{n} \bar{u}_{j}^{n}\right)_{\widehat{x}}\right]=0, \\
\left(\rho_{j}^{n}\right)_{\widehat{t}}+\left(u_{j}^{n}\right)_{\widehat{x}}+r \bar{\rho}_{j}^{n}=0, \\
u_{j}^{0}=u_{0}\left(x_{j}\right), \quad \rho_{j}^{0}=\rho_{0}\left(x_{j}\right), \quad 0 \leq j \leq J, \\
u_{0}^{n}=u_{J}^{n}=0, \quad \rho_{0}^{n}=\rho_{J}^{n}=0, \quad 1 \leq n \leq N .
\end{gathered}
$$

Lemma 2.1. Summation by parts follows $[12,21]$ that for any two discrete functions $u, v \in Z_{h}^{0}$

$$
\left\langle\left(u_{j}\right)_{x^{\prime}} v_{j}\right\rangle=-\left\langle u_{j},\left(v_{j}\right)_{\bar{x}}\right\rangle, \quad\left\langle v_{j},\left(u_{j}\right)_{x \bar{x}}\right\rangle=-\left\langle\left(v_{j}\right)_{x^{\prime}}\left(u_{j}\right)_{x}\right\rangle .
$$


Lemma 2.2 (discrete Sobolev's inequality $[12,21]$ ). There exist two constants $C_{1}$ and $C_{2}$ such that

$$
\left\|u^{n}\right\|_{\infty} \leq C_{1}\left\|u^{n}\right\|+C_{2}\left\|u_{x}^{n}\right\|
$$

Lemma 2.3 (discrete Gronwall inequality $[12,21]$ ). Suppose that $w(k), \rho(k)$ are nonnegative functions and $\rho(k)$ is nondecreasing. If $C>0$ and

$$
w(k) \leq \rho(k)+C \tau \sum_{l=0}^{k-1} w(l) .
$$

Then $w(k) \leq \rho(k) e^{C \tau k}$.

Theorem 2.4. If $u_{0} \in H^{1}, \rho_{0} \in L_{2}$, then the solution of (2.2)-(2.5) satisfies

$$
\left\|u^{n}\right\| \leq C, \quad\left\|u_{x}^{n}\right\| \leq C, \quad\left\|\rho^{n}\right\| \leq C, \quad\left\|u^{n}\right\|_{\infty} \leq C \quad(n=1,2, \ldots, N) .
$$

Proof. Taking an inner product of (2.2) with $2 \bar{u}_{j}^{n}$ (i.e., $u_{j}^{n+1}+u_{j}^{n-1}$ ) and considering the boundary condition (2.5) and Lemma 2.1, we obtain

$$
\begin{aligned}
& \frac{1}{2 \tau}\left(\left\|u^{n+1}\right\|^{2}-\left\|u^{n-1}\right\|^{2}\right)+\frac{1}{2 \tau}\left(\left\|u_{x}^{n+1}\right\|^{2}-\left\|u_{x}^{n-1}\right\|^{2}\right) \\
& \quad+\left\langle\left(\rho_{j}^{n}\right)_{\widehat{x}^{\prime}} 2 \bar{u}_{j}^{n}\right\rangle-v\left\langle\left(\bar{u}_{j}^{n}\right)_{x \bar{x}^{\prime}} 2 \bar{u}_{j}^{n}\right\rangle+\left\langle P, 2 \bar{u}_{j}^{n}\right\rangle=0,
\end{aligned}
$$

where $P=(1 / 3)\left[u_{j}^{n}\left(\bar{u}_{j}^{n}\right)_{\widehat{x}}+\left(u_{j}^{n} \bar{u}_{j}^{n}\right)_{\widehat{x}}\right]$. Since

$$
\begin{gathered}
\left\langle\left(\rho_{j}^{n}\right)_{\widehat{x}^{\prime}} 2 \bar{u}_{j}^{n}\right\rangle=-\left\langle\rho_{j}^{n}, 2\left(\bar{u}_{j}^{n}\right)_{\hat{x}}\right\rangle, \\
\left\langle\left(\bar{u}_{j}^{n}\right)_{x \bar{x}^{\prime}} 2 \bar{u}_{j}^{n}\right\rangle=-2\left\|\bar{u}_{x}^{n}\right\|^{2}, \\
\left\langle P, 2 \bar{u}_{j}^{n}\right\rangle=\frac{2}{3} h \sum_{j=0}^{J-1}\left[u_{j}^{n}\left(\bar{u}_{j}^{n}\right)_{\widehat{x}}+\left(u_{j}^{n} \bar{u}_{j}^{n}\right)_{\hat{x}} \bar{u}_{j}^{n}\right. \\
=\frac{1}{12} \sum_{j=0}^{J-1}\left[u_{j}^{n}\left(u_{j+1}^{n+1}+u_{j+1}^{n-1}-u_{j-1}^{n+1}-u_{j-1}^{n-1}\right)+u_{j+1}^{n}\left(u_{j+1}^{n+1}+u_{j+1}^{n-1}\right)-u_{j-1}^{n}\left(u_{j-1}^{n+1}+u_{j-1}^{n-1}\right)\right] \\
\times\left(u_{j}^{n+1}+u_{j}^{n-1}\right) \\
=\frac{1}{12} \sum_{j=0}^{J-1}\left(u_{j}^{n}+u_{j+1}^{n}\right)\left(u_{j+1}^{n+1}+u_{j+1}^{n-1}\right)\left(u_{j}^{n+1}+u_{j}^{n-1}\right) \\
-\frac{1}{12} \sum_{j=0}^{J-1}\left(u_{j}^{n}+u_{j-1}^{n}\right)\left(u_{j}^{n+1}+u_{j}^{n-1}\right)\left(u_{j-1}^{n+1}+u_{j-1}^{n-1}\right)=0,
\end{gathered}
$$


we obtain

$$
\frac{1}{2 \tau}\left(\left\|u^{n+1}\right\|^{2}-\left\|u^{n-1}\right\|^{2}\right)+\frac{1}{2 \tau}\left(\left\|u_{x}^{n+1}\right\|^{2}-\left\|u_{x}^{n-1}\right\|^{2}\right)-\left\langle\rho_{j}^{n}, 2\left(\bar{u}_{j}^{n}\right)_{\hat{x}}\right\rangle+2 v\left\|\bar{u}_{x}^{n}\right\|^{2}=0 .
$$

Taking an inner product of (2.3) with $2 \bar{\rho}_{j}^{n}$ (i.e., $\rho_{j}^{n+1}+\rho_{j}^{n-1}$ ), we obtain

$$
\frac{1}{2 \tau}\left(\left\|\rho^{n+1}\right\|^{2}-\left\|\rho^{n-1}\right\|^{2}\right)+\left\langle\left(u_{j}^{n}\right)_{\widehat{x}^{\prime}} 2 \bar{\rho}_{j}^{n}\right\rangle+2 \gamma\left\|\bar{\rho}_{j}^{n}\right\|^{2}=0
$$

Adding (2.12) to (2.13), we have

$$
\begin{aligned}
& \left\|u^{n+1}\right\|^{2}-\left\|u^{n-1}\right\|^{2}+\left\|u_{x}^{n+1}\right\|^{2}-\left\|u_{x}^{n-1}\right\|^{2}+\left\|\rho^{n+1}\right\|^{2}-\left\|\rho^{n-1}\right\|^{2} \\
& \quad=2 \tau\left[\left\langle\rho_{j}^{n}, 2\left(\bar{u}_{j}^{n}\right)_{\widehat{x}}\right\rangle-\left\langle\left(u_{j}^{n}\right)_{\widehat{x}^{\prime}} 2 \bar{\rho}_{j}^{n}\right\rangle\right]-4 v \tau\left\|\bar{u}_{x}^{n}\right\|^{2}-4 \gamma \tau\left\|\bar{\rho}_{j}^{n}\right\|^{2} .
\end{aligned}
$$

Since

$$
\begin{aligned}
& \left\langle\rho_{j}^{n}, 2\left(\bar{u}_{j}^{n}\right)_{\widehat{x}}\right\rangle=\left\langle\rho_{j}^{n},\left(u_{j}^{n+1}\right)_{\hat{x}}+\left(u_{j}^{n-1}\right)_{\hat{x}}\right\rangle \leq\left\|\rho^{n}\right\|^{2}+\frac{1}{2}\left(\left\|u_{x}^{n+1}\right\|^{2}+\left\|u_{x}^{n-1}\right\|^{2}\right), \\
& -\left\langle\left(u_{j}^{n}\right)_{\widehat{x}^{\prime}} 2 \bar{\rho}_{j}^{n}\right\rangle=-\left\langle\left(u_{j}^{n}\right)_{\widehat{x}^{\prime}} \rho_{j}^{n+1}+\rho_{j}^{n-1}\right\rangle \leq\left\|u_{x}^{n}\right\|^{2}+\frac{1}{2}\left(\left\|\rho^{n+1}\right\|^{2}+\left\|\rho^{n-1}\right\|^{2}\right) .
\end{aligned}
$$

Equation (2.14) can be changed to

$$
\begin{aligned}
& \left\|u^{n+1}\right\|^{2}-\left\|u^{n-1}\right\|^{2}+\left\|u_{x}^{n+1}\right\|^{2}-\left\|u_{x}^{n-1}\right\|^{2}+\left\|\rho^{n+1}\right\|^{2}-\left\|\rho^{n-1}\right\|^{2} \\
& \quad \leq C \tau\left(\left\|u_{x}^{n+1}\right\|^{2}+\left\|u_{x}^{n}\right\|^{2}+\left\|u_{x}^{n-1}\right\|^{2}+\left\|\rho^{n+1}\right\|^{2}+\left\|\rho^{n}\right\|^{2}+\left\|\rho^{n-1}\right\|^{2}\right) .
\end{aligned}
$$

Let $A^{n}=\left\|u^{n+1}\right\|^{2}+\left\|u^{n}\right\|^{2}+\left\|u_{x}^{n+1}\right\|^{2}+\left\|u_{x}^{n}\right\|^{2}+\left\|\rho^{n+1}\right\|^{2}+\left\|\rho^{n}\right\|^{2}$, and (2.16) is changed to

$$
A^{n}-A^{n-1} \leq C \tau\left(A^{n}+A^{n-1}\right)
$$

If $\tau$ is sufficiently small which satisfies $1-C \tau>0$, then

$$
A^{n}-A^{n-1} \leq C \tau A^{n-1} .
$$

Summing up (2.18) from 1 to $n$, we have

$$
A^{n} \leq A^{0}+C \tau \sum_{l=0}^{n-1} A^{l}
$$


From Lemma 2.3, we obtain $A^{n} \leq C$, which implies that, $\left\|u^{n}\right\| \leq C,\left\|u_{x}^{n}\right\| \leq C$, and $\left\|\rho^{n}\right\| \leq C$. By Lemma 2.2, we obtain $\left\|u^{n}\right\|_{\infty} \leq C$.

Theorem 2.5. Assume that $u^{0} \in H^{2}, \rho^{0} \in H^{1}$, the solution of difference scheme (2.2)-(2.5) satisfies:

$$
\left\|\rho_{x}^{n}\right\| \leq C, \quad\left\|u_{x x}^{n}\right\| \leq C, \quad\left\|u_{x}^{n}\right\|_{\infty} \leq C, \quad\left\|\rho^{n}\right\|_{\infty} \leq C \quad(n=1,2, \ldots, N) .
$$

Proof. Differentiating backward (2.2)-(2.5) with respect to $x$, we obtain

$$
\begin{gathered}
\left(u_{j}^{n}\right)_{x \widehat{t}}-\left(u_{j}^{n}\right)_{x x \bar{x} \hat{t}}+\left(\rho_{j}^{n}\right)_{x \widehat{x}}-v\left(\bar{u}_{j}^{n}\right)_{x x \bar{x}}+\frac{1}{3}\left[u_{j}^{n}\left(\bar{u}_{j}^{n}\right)_{\widehat{x}}+\left(u_{j}^{n} \bar{u}_{j}^{n}\right)_{\widehat{x}}\right]_{x}=0, \\
\left(\rho_{j}^{n}\right)_{x \widehat{t}}+\left(u_{j}^{n}\right)_{x \hat{x}}+r\left(\bar{\rho}_{j}^{n}\right)_{x}=0, \\
\left(u_{j}^{0}\right)_{x}=u_{0, x}\left(x_{j}\right), \quad\left(\rho_{j}^{0}\right)_{x}=\rho_{0, x}\left(x_{j}\right), \quad 0 \leq j \leq J, \\
\left(u_{0}^{n}\right)_{x}=\left(u_{J}^{n}\right)_{x}=0, \quad\left(\rho_{0}^{n}\right)_{x}=\left(\rho_{J}^{n}\right)_{x}=0, \quad 0 \leq n \leq N .
\end{gathered}
$$

Computing the inner product of (2.21) with $2 \bar{u}_{x}^{n}$ (i.e., $u_{x}^{n+1}+u_{x}^{n-1}$ ) and considering (2.24) and Lemma 2.1, we obtain

$$
\begin{gathered}
\frac{1}{2 \tau}\left(\left\|u_{x}^{n+1}\right\|^{2}-\left\|u_{x}^{n-1}\right\|^{2}\right)+\frac{1}{2 \tau}\left(\left\|u_{x x}^{n+1}\right\|^{2}-\left\|u_{x x}^{n-1}\right\|^{2}\right) \\
+\left\langle\rho_{x x^{\prime}}^{n} 2 \bar{u}_{x}^{n}\right\rangle-v\left\langle\bar{u}_{x x \bar{x}}^{n}, 2 \bar{u}_{x}^{n}\right\rangle+\left\langle R, 2 \bar{u}_{x}^{n}\right\rangle=0
\end{gathered}
$$

where $R=(1 / 3)\left[\left(u_{j}^{n}\left(\bar{u}_{j}^{n}\right)_{\hat{x}}+\left(u_{j}^{n} \bar{u}_{j}^{n}\right)_{\widehat{x}}\right]_{x}\right.$. It follows from Theorem 2.4 that

$$
\left|u_{j}^{n}\right| \leq C \quad(j=0,1,2, \ldots, J)
$$

By the Schwarz inequality and Lemma 2.1, we get

$$
\begin{aligned}
\left\langle R, 2 \bar{u}_{x}^{n}\right\rangle & =\frac{2}{3}\left\langle\left[u_{j}^{n}\left(\bar{u}_{j}^{n}\right)_{\widehat{x}}+\left(u_{j}^{n} \bar{u}_{j}^{n}\right)_{\widehat{x}}\right]_{x}, \bar{u}_{x}^{n}\right\rangle \\
& =-\frac{2}{3}\left\langle u_{j}^{n}\left(\bar{u}_{j}^{n}\right)_{\widehat{x}}+\left(u_{j}^{n} \bar{u}_{j}^{n}\right)_{\widehat{x}}, \bar{u}_{x \bar{x}}^{n}\right\rangle \\
& =-\frac{2}{3} h \sum_{j=0}^{J-1}\left[u_{j}^{n}\left(\bar{u}_{j}^{n}\right)_{\widehat{x}}+\left(u_{j}^{n} \bar{u}_{j}^{n}\right)_{\widehat{x}}\right]\left(\bar{u}_{j}^{n}\right)_{x \bar{x}} \\
& \leq \frac{2}{3} C h \sum_{j=0}^{J-1}\left|\left(\bar{u}_{j}^{n}\right)_{\widehat{x}}\right| \cdot\left|\left(\bar{u}_{j}^{n}\right)_{x \bar{x}}\right| \leq C\left(\left\|\bar{u}_{x}^{n}\right\|^{2}+\left\|\bar{u}_{x x}^{n}\right\|^{2}\right) \\
& \leq C\left(\left\|u_{x}^{n+1}\right\|^{2}+\left\|u_{x}^{n-1}\right\|^{2}+\left\|u_{x x}^{n+1}\right\|^{2}+\left\|u_{x x}^{n-1}\right\|^{2}\right) .
\end{aligned}
$$


Noting that

$$
\begin{gathered}
\left\langle\rho_{x \hat{x}}^{n}, 2 \bar{u}_{x}^{n}\right\rangle=-\left\langle 2 \bar{u}_{x \hat{x}}^{n}, \rho_{x}^{n}\right\rangle=-\left\langle\rho_{x}^{n}, u_{x \hat{x}}^{n+1}+u_{x \hat{x}}^{n-1}\right\rangle \leq\left\|\rho_{x}^{n}\right\|^{2}+\frac{1}{2}\left(\left\|u_{x x}^{n+1}\right\|^{2}+\left\|u_{x x}^{n-1}\right\|^{2}\right), \\
\left\langle\bar{u}_{x x \bar{x},}^{n}, 2 \bar{u}_{x}^{n}\right\rangle=-2\left\|\bar{u}_{x x}^{n}\right\|^{2},
\end{gathered}
$$

it follows from (2.25) that

$$
\begin{aligned}
& \left\|u_{x}^{n+1}\right\|^{2}-\left\|u_{x}^{n-1}\right\|^{2}+\left\|u_{x x}^{n+1}\right\|^{2}-\left\|u_{x x}^{n-1}\right\|^{2} \\
& \quad \leq-4 v \tau\left\|\bar{u}_{x x}^{n}\right\|^{2}+C \tau\left(\left\|u_{x}^{n+1}\right\|^{2}+\left\|u_{x}^{n-1}\right\|^{2}+\left\|u_{x x}^{n+1}\right\|^{2}+\left\|u_{x x}^{n-1}\right\|^{2}+\left\|\rho_{x}^{n}\right\|^{2}\right) .
\end{aligned}
$$

Computing the inner product of (2.22) with $2 \bar{\rho}_{x}^{n}$ (i.e., $\rho_{x}^{n+1}+\rho_{x}^{n-1}$ ) and considering (2.24) and Lemma 2.1, we obtain

$$
\frac{1}{2 \tau}\left(\left\|\rho_{x}^{n+1}\right\|^{2}-\left\|\rho_{x}^{n-1}\right\|^{2}\right)+\left\langle u_{x \widehat{x}}^{n}, \bar{\rho}_{x}^{n}\right\rangle+2 \gamma\left\|\bar{\rho}_{x}^{n}\right\|^{2}=0
$$

Since

$$
\left\langle u_{x \widehat{x}^{\prime}}^{n}, 2 \bar{\rho}_{x}^{n}\right\rangle=\left\langle u_{x x^{\prime}}^{n} \rho_{x}^{n+1}+\rho_{x}^{n-1}\right\rangle \leq\left\|u_{x x}^{n}\right\|^{2}+\frac{1}{2}\left(\left\|\rho_{x}^{n+1}\right\|^{2}+\left\|\rho_{x}^{n-1}\right\|^{2}\right)
$$

then (2.30) is changed to

$$
\left\|\rho_{x}^{n+1}\right\|-\left\|\rho_{x}^{n-1}\right\| \leq-4 \gamma \tau\left\|\bar{\rho}_{x}^{n}\right\|^{2}+C \tau\left(\left\|u_{x x}^{n}\right\|^{2}+\left\|\rho_{x}^{n+1}\right\|^{2}+\left\|\rho_{x}^{n-1}\right\|^{2}\right) .
$$

Adding (2.29) to (2.32), we have

$$
\begin{aligned}
& \left\|u_{x}^{n+1}\right\|^{2}-\left\|u_{x}^{n-1}\right\|^{2}+\left\|u_{x x}^{n+1}\right\|^{2}-\left\|u_{x x}^{n-1}\right\|^{2}+\left\|\rho_{x}^{n+1}\right\|^{2}-\left\|\rho_{x}^{n-1}\right\|^{2} \\
& \leq-4 v \tau\left\|\bar{u}_{x x}^{n}\right\|^{2}-4 \gamma \tau\left\|\bar{\rho}_{x}^{n}\right\|^{2} \\
& \quad+C \tau\left(\left\|u_{x}^{n+1}\right\|^{2}+\left\|u_{x}^{n-1}\right\|^{2}+\left\|u_{x x}^{n}\right\|^{2}+\left\|u_{x x}^{n+1}\right\|^{2}+\left\|u_{x x}^{n-1}\right\|^{2}+\left\|\rho_{x}^{n+1}\right\|^{2}+\left\|\rho_{x}^{n}\right\|^{2}+\left\|\rho_{x}^{n-1}\right\|^{2}\right) \\
& \leq C \tau\left(\left\|u_{x}^{n+1}\right\|^{2}+\left\|u_{x}^{n-1}\right\|^{2}+\left\|u_{x x}^{n}\right\|^{2}+\left\|u_{x x}^{n+1}\right\|^{2}+\left\|u_{x x}^{n-1}\right\|^{2}+\left\|\rho_{x}^{n+1}\right\|^{2}+\left\|\rho_{x}^{n}\right\|^{2}+\left\|\rho_{x}^{n-1}\right\|^{2}\right) .
\end{aligned}
$$

Leting $B^{n}=\left\|u_{x}^{n+1}\right\|^{2}+\left\|u_{x}^{n}\right\|^{2}+\left\|u_{x x}^{n+1}\right\|^{2}+\left\|u_{x x}^{n}\right\|^{2}+\left\|\rho_{x}^{n+1}\right\|^{2}+\left\|\rho_{x}^{n}\right\|^{2}$, we obtain $B^{n}-B^{n-1} \leq C \tau\left(B^{n}+\right.$ $\left.B^{n-1}\right)$. Choosing suitable $\tau$ which is small enough to satisfy $1-C \tau>0$, we get

$$
B^{n}-B^{n-1} \leq C \tau B^{n-1} .
$$


Summing up (2.34) from 1 to $n$, we have

$$
B^{n} \leq B^{0}+C \tau \sum_{l=0}^{n-1} B^{l}
$$

By Lemma 2.3, we get $B^{n} \leq C$, which implies that $\left\|\rho_{x}^{n}\right\| \leq C,\left\|u_{x x}^{n}\right\| \leq C$. It follows from Theorem 2.4 and Lemma 2.2 that $\left\|u_{x}^{n}\right\|_{\infty} \leq C,\left\|\rho^{n}\right\|_{\infty} \leq C$.

\section{Solvability}

Theorem 3.1. The solution $u^{n}$ of (2.2)-(2.5) is unique.

Proof. Using the mathematical induction, clearly, $u^{0}, \rho^{0}$ are uniquely determined by initial conditions (2.4). then select appropriate second-order methods (such as the C-N Schemes) and calculate $u^{1}$ and $\rho^{1}$ (i.e. $u^{0}, \rho^{0}$, and $u^{1}, \rho^{1}$ are uniquely determined). Assume that $u^{0}, u^{1}, \ldots, u^{n}$ and $\rho^{0}, \rho^{1}, \ldots, \rho^{n}$ are the only solution, now consider $u^{n+1}$ and $\rho^{n+1}$ in (2.2) and (2.3):

$$
\begin{gathered}
\frac{1}{2 \tau} u_{j}^{n+1}-\frac{1}{2 \tau}\left(u_{j}^{n+1}\right)_{x \bar{x}}-\frac{v}{2}\left(u_{j}^{n+1}\right)_{x \bar{x}}+\frac{1}{6}\left[u_{j}^{n}\left(u_{j}^{n+1}\right)_{\widehat{x}}+\left(u_{j}^{n} u_{j}^{n+1}\right)_{\widehat{x}}\right]=0 \\
\frac{1}{2 \tau} \rho_{j}^{n+1}+\frac{\gamma}{2} \rho_{j}^{n+1}=0
\end{gathered}
$$

Taking an inner product of (3.1) with $u^{n+1}$, we have

$$
\frac{1}{2 \tau}\left\|u^{n+1}\right\|^{2}+\frac{1}{2 \tau}\left\|u_{x}^{n+1}\right\|^{2}+\frac{v}{2}\left\|u_{x}^{n+1}\right\|^{2}+\frac{1}{6} h \sum_{j=0}^{J-1}\left[u_{j}^{n}\left(u_{j}^{n+1}\right)_{\widehat{x}}+\left(u_{j}^{n} u_{j}^{n+1}\right)_{\widehat{x}}\right] u_{j}^{n+1}=0
$$

Since

$$
\begin{aligned}
\frac{1}{6} & h \sum_{j=0}^{J-1}\left[u_{j}^{n}\left(u_{j}^{n+1}\right)_{\widehat{x}}+\left(u_{j}^{n} u_{j}^{n+1}\right)_{\widehat{x}}\right] u_{j}^{n+1} \\
& =\frac{1}{12} \sum_{j=0}^{J-1}\left[u_{j}^{n}\left(u_{j+1}^{n+1}-u_{j-1}^{n+1}\right)+\left(u_{j+1}^{n} u_{j+1}^{n+1}-u_{j-1}^{n} u_{j-1}^{n+1}\right)\right] u_{j}^{n+1} \\
& =\frac{1}{12} \sum_{j=0}^{J-1}\left[u_{j}^{n} u_{j}^{n+1} u_{j+1}^{n+1}+u_{j+1}^{n} u_{j}^{n+1} u_{j+1}^{n+1}\right]-\frac{1}{12} \sum_{j=0}^{J-1}\left[u_{j-1}^{n} u_{j-1}^{n+1} u_{j}^{n+1}+u_{j}^{n} u_{j-1}^{n+1} u_{j}^{n+1}\right]=0,
\end{aligned}
$$

then it holds

$$
\frac{1}{2 \tau}\left\|u^{n+1}\right\|^{2}+\left(\frac{1}{2 \tau}+\frac{v}{2}\right)\left\|u_{x}^{n+1}\right\|^{2}=0
$$


Taking an inner product of (3.2) with $\rho^{n+1}$ and adding to (3.5), we have

$$
\frac{1}{2 \tau}\left\|u^{n+1}\right\|^{2}+\left(\frac{1}{2 \tau}+\frac{v}{2}\right)\left\|u_{x}^{n+1}\right\|^{2}+\left(\frac{1}{2 \tau}+\frac{\gamma}{2}\right)\left\|\rho^{n+1}\right\|^{2}=0
$$

which implies that (3.1)-(3.2) have only zero solution. So the solution $u_{j}^{n+1}$ and $\rho_{j}^{n+1}$ of (2.2)(2.5) is unique.

\section{Convergence and Stability}

Let $v(x, t)$ and $\emptyset(x, t)$ be the solution of problem (1.4)-(1.7); that is, $v_{j}^{n}=u\left(x_{j}, t_{n}\right), \emptyset_{j}^{n}=$ $\rho\left(x_{j}, t_{n}\right)$, then the truncation of the difference scheme (2.2)-(2.5) is

$$
\begin{gathered}
r_{j}^{n}=\left(v_{j}^{n}\right)_{\widehat{t}}-\left(v_{j}^{n}\right)_{x \bar{x} \hat{t}}+\left(\emptyset_{j}^{n}\right)_{\widehat{x}}-v\left(\bar{v}_{j}^{n}\right)_{x \bar{x}}+\frac{1}{3}\left[v_{j}^{n}\left(\bar{v}_{j}^{n}\right)_{\widehat{x}}+\left(v_{j}^{n} \bar{v}_{j}^{n}\right)_{\widehat{x}}\right], \\
s_{j}^{n}=\left(\emptyset_{j}^{n}\right)_{\widehat{t}}+\left(v_{j}^{n}\right)_{\widehat{x}}+\gamma \bar{\emptyset}_{j}^{n} .
\end{gathered}
$$

Making use of Taylor expansion, it holds $\left|r_{j}^{n}\right|+\left|s_{j}^{n}\right|=O\left(\tau^{2}+h^{2}\right)$ if $h, \tau \rightarrow 0$.

Theorem 4.1. Assume that $u_{0} \in H^{1}, \rho_{0} \in L_{2}$, then the solution $u^{n}$ and $\rho^{n}$ in the senses of norms $\|\cdot\|_{\infty}$ and $\|\cdot\|_{L^{2}}$, respectively, to the difference scheme (2.2)-(2.5) converges to the solution of problem (1.4)-(1.7) and the order of convergence is $O\left(\tau^{2}+h^{2}\right)$.

Proof. Subtracting (2.2) from (4.1) subtracting (2.3) from (4.2), and letting $e_{j}^{n}=v_{j}^{n}-u_{j}^{n}, \eta_{j}^{n}=$ $\emptyset_{j}^{n}-\rho_{j}^{n}$, we have

$$
\begin{gathered}
r_{j}^{n}=\left(e_{j}^{n}\right)_{\widehat{t}}-\left(e_{j}^{n}\right)_{x \widehat{x} \hat{t}}+\left(\eta_{j}^{n}\right)_{\widehat{x}}-v\left(\bar{e}_{j}^{n}\right)_{x \bar{x}}+Q, \\
s_{j}^{n}=\left(\eta_{j}^{n}\right)_{\widehat{t}}+\left(e_{j}^{n}\right)_{\widehat{x}}+\gamma \bar{\eta}_{j}^{n},
\end{gathered}
$$

where

$$
Q=\frac{1}{3}\left[v_{j}^{n}\left(\bar{v}_{j}^{n}\right)_{\widehat{x}}-u_{j}^{n}\left(\bar{u}_{j}^{n}\right)_{\widehat{x}}\right]+\frac{1}{3}\left[\left(v_{j}^{n} \bar{v}_{j}^{n}\right)_{\widehat{x}}-\left(u_{j}^{n} \bar{u}_{j}^{n}\right)_{\widehat{x}}\right] .
$$

Computing the inner product of (4.3) with $2 \bar{e}^{n}$, we get

$$
\begin{gathered}
\left\|e^{n+1}\right\|^{2}-\left\|e^{n-1}\right\|^{2}+\left\|e_{x}^{n+1}\right\|^{2}-\left\|e_{x}^{n-1}\right\|^{2}=-4 v \tau\left\|\bar{e}_{x}^{n}\right\|^{2} \\
+2 \tau\left[\left\langle r_{j}^{n}, 2 \bar{e}_{j}^{n}\right\rangle-\left\langle\left(\eta_{j}^{n}\right)_{\widehat{x}^{\prime}} 2 \bar{e}_{j}^{n}\right\rangle-\left\langle Q, 2 \bar{e}_{j}^{n}\right\rangle\right] .
\end{gathered}
$$


According to

$$
\begin{aligned}
-\left\langle Q, 2 \bar{e}_{j}^{n}\right\rangle & =-\frac{2}{3} h \sum_{j=0}^{J-1}\left[v_{j}^{n}\left(\bar{v}_{j}^{n}\right)_{\widehat{x}}-u_{j}^{n}\left(\bar{u}_{j}^{n}\right)_{\widehat{x}}\right] \bar{e}_{j}^{n}-\frac{2}{3} h \sum_{j=0}^{J-1}\left[\left(v_{j}^{n} \bar{v}_{j}^{n}\right)_{\widehat{x}}-\left(u_{j}^{n} \bar{u}_{j}^{n}\right)_{\widehat{x}}\right] \bar{e}_{j}^{n} \\
& =-\frac{2}{3} h \sum_{j=0}^{J-1}\left[v_{j}^{n}\left(\bar{e}_{j}^{n}\right)_{\widehat{x}}+e_{j}^{n}\left(\bar{u}_{j}^{n}\right)_{\widehat{x}}\right] \bar{e}_{j}^{n}+\frac{2}{3} h \sum_{j=0}^{J-1}\left[v_{j}^{n} \bar{v}_{j}^{n}-u_{j}^{n} \bar{u}_{j}^{n}\right]\left(\bar{e}_{j}^{n}\right)_{\widehat{x}} \\
& =-\frac{2}{3} h \sum_{j=0}^{J-1}\left[v_{j}^{n}\left(\bar{e}_{j}^{n}\right)_{\widehat{x}}+e_{j}^{n}\left(\bar{u}_{j}^{n}\right)_{\widehat{x}}\right] \bar{e}_{j}^{n}+\frac{2}{3} h \sum_{j=0}^{J-1}\left[e_{j}^{n} \bar{v}_{j}^{n}+u_{j}^{n} \bar{e}_{j}^{n}\right]\left(\bar{e}_{j}^{n}\right)_{\hat{x}^{\prime}}
\end{aligned}
$$

it follow from Lemma 1.1, Theorems 2.4, and 2.5 that

$$
\left|v_{j}^{n}\right| \leq C, \quad\left|\bar{v}_{j}^{n}\right| \leq C, \quad\left|\left(\bar{u}_{j}^{n}\right)_{\widehat{x}}\right| \leq C, \quad\left|u_{j}^{n}\right| \leq C \quad(j=0,1,2, \ldots, J) .
$$

By the Schwarz inequality, we obtain

$$
\begin{aligned}
-\left\langle Q, 2 \bar{e}^{n}\right\rangle & \leq \frac{2}{3} C h \sum_{j=0}^{J-1}\left(\left|\left(\bar{e}_{j}^{n}\right)_{\hat{x}}\right|+\left|e_{j}^{n}\right|\right) \cdot\left|\bar{e}_{j}^{n}\right|+\frac{2}{3} C h \sum_{j=0}^{J-1}\left(\left|e_{j}^{n}\right|+\left|\bar{e}_{j}^{n}\right|\right) \cdot\left|\left(\bar{e}_{j}^{n}\right)_{\hat{x}}\right| \\
& \leq C\left(\left\|\bar{e}_{x}^{n}\right\|^{2}+\left\|e^{n}\right\|^{2}+\left\|\bar{e}^{n}\right\|^{2}\right) \\
& \leq C\left(\left\|e^{n+1}\right\|^{2}+\left\|e^{n}\right\|^{2}+\left\|e^{n-1}\right\|^{2}+\left\|e_{x}^{n+1}\right\|^{2}+\left\|e_{x}^{n-1}\right\|^{2}\right) .
\end{aligned}
$$

Since

$$
\begin{gathered}
\left\langle r_{j}^{n}, 2 \bar{e}_{j}^{n}\right\rangle=\left\langle r_{j}^{n}, e_{j}^{n+1}+e_{j}^{n-1}\right\rangle \leq\left\|r^{n}\right\|^{2}+\frac{1}{2}\left(\left\|e^{n+1}\right\|^{2}+\left\|e^{n-1}\right\|^{2}\right), \\
-\left\langle\left(\eta_{j}^{n}\right)_{\widehat{x}^{\prime}} 2 \bar{e}_{j}^{n}\right\rangle=\left\langle\eta_{j}^{n}, 2\left(\bar{e}_{j}^{n}\right)_{\hat{x}}\right\rangle \leq\left\|\eta^{n}\right\|^{2}+\frac{1}{2}\left[\left\|e_{x}^{n+1}\right\|^{2}+\left\|e_{x}^{n-1}\right\|^{2}\right],
\end{gathered}
$$

it follows from (4.9)-(4.10) and (4.6) that

$$
\begin{aligned}
& \left\|e^{n+1}\right\|^{2}-\left\|e^{n-1}\right\|^{2}+\left\|e_{x}^{n+1}\right\|^{2}-\left\|e_{x}^{n-1}\right\|^{2} \\
& \quad \leq 2 \tau\left\|r^{n}\right\|+C \tau\left(\left\|e^{n+1}\right\|^{2}+\left\|e^{n}\right\|^{2}+\left\|e^{n-1}\right\|^{2}+\left\|e_{x}^{n+1}\right\|^{2}+\left\|e_{x}^{n-1}\right\|^{2}+\|\eta\|^{2}\right) .
\end{aligned}
$$


Computing the inner product of (4.4) with $2 \bar{\eta}^{n}$, we obtain

$$
\begin{aligned}
\left\|\eta^{n+1}\right\|^{2}-\left\|\eta^{n-1}\right\|^{2} & =2 \tau\left\langle s_{j}^{n}, 2 \bar{\eta}_{j}^{n}\right\rangle-2 \tau\left\langle\left(e_{j}^{n}\right)_{\widehat{x}^{\prime}} 2 \bar{\eta}_{j}^{n}\right\rangle-2 \gamma \tau\left\|\bar{\eta}^{n}\right\|^{2} \\
& \leq C \tau\left(\left\|\eta^{n+1}\right\|^{2}+\left\|\eta^{n-1}\right\|^{2}\left\|e_{x}^{n}\right\|^{2}\right)+2 \tau\left\|s^{n}\right\|^{2} .
\end{aligned}
$$

Adding (4.12) to (4.11), we have

$$
\begin{aligned}
&\left\|e^{n+1}\right\|^{2}-\left\|e^{n-1}\right\|^{2}+\left\|e_{x}^{n+1}\right\|^{2}-\left\|e_{x}^{n-1}\right\|^{2}+\left\|\eta^{n+1}\right\|^{2}-\left\|\eta^{n-1}\right\|^{2} \\
& \leq 2 \tau\left\|r^{n}\right\|^{2}+2 \tau\left\|s^{n}\right\|^{2}+C \tau\left[\left\|e^{n+1}\right\|^{2}+\left\|e^{n}\right\|^{2}+\left\|e^{n-1}\right\|^{2}+\left\|e_{x}^{n+1}\right\|^{2}\right. \\
&\left.+\left\|e_{x}^{n-1}\right\|^{2}+\left\|e_{x}^{n}\right\|^{2}+\left\|\eta^{n+1}\right\|^{2}+\left\|\eta^{n}\right\|^{2}+\left\|\eta^{n-1}\right\|^{2}\right] .
\end{aligned}
$$

Leting

$$
D^{n}=\left\|e^{n}\right\|^{2}+\left\|e^{n+1}\right\|^{2}+\left\|e_{x}^{n}\right\|^{2}+\left\|e_{x}^{n+1}\right\|^{2}+\left\|\eta^{n}\right\|^{2}+\left\|\eta^{n+1}\right\|^{2}
$$

we get

$$
D^{n}-D^{n-1} \leq 2 \tau\left\|r^{n}\right\|^{2}+2 \tau\left\|s^{n}\right\|^{2}+C \tau\left(D^{n+1}+D^{n}\right) .
$$

If $\tau$ is sufficiently small which satisfies $1-C \tau>0$, then

$$
D^{n}-D^{n-1} \leq C \tau D^{n-1}+C \tau\left\|r^{n}\right\|^{2}+C \tau\left\|s^{n}\right\|^{2}
$$

Summing up (4.16) from 1 to $n$, we have

$$
D^{n} \leq D^{0}+C \tau \sum_{l=1}^{n}\left\|r^{l}\right\|^{2}+C \tau \sum_{l=1}^{n}\left\|s^{l}\right\|^{2}+C \tau \sum_{l=0}^{n-1} D^{l} .
$$

Select appropriate second-order methods (such as the C-N Schemes), and calculate $u^{1}$ and $\rho^{1}$, which satisfies

$$
D^{0}=O\left(\tau^{2}+h^{2}\right)^{2}
$$


Noticing that

$$
\begin{aligned}
& \tau \sum_{l=1}^{n}\left\|r^{l}\right\|^{2} \leq n \tau \max _{1 \leq l \leq n}\left\|r^{l}\right\|^{2} \leq T \cdot O\left(\tau^{2}+h^{2}\right)^{2}, \\
& \tau \sum_{l=1}^{n}\left\|s^{l}\right\|^{2} \leq n \tau \max _{1 \leq l \leq n}\left\|s^{l}\right\|^{2} \leq T \cdot O\left(\tau^{2}+h^{2}\right)^{2},
\end{aligned}
$$

we then have

$$
D^{n} \leq O\left(\tau^{2}+h^{2}\right)^{2}+C \tau \sum_{l=0}^{n-1} D^{l}
$$

By Lemma 2.3, we get

$$
D^{n} \leq O\left(\tau^{2}+h^{2}\right)^{2}
$$

This yields

$$
\left\|e^{n}\right\| \leq O\left(\tau^{2}+h^{2}\right), \quad\left\|e_{x}^{n}\right\| \leq O\left(\tau^{2}+h^{2}\right), \quad\left\|\eta^{n}\right\| \leq O\left(\tau^{2}+h^{2}\right) .
$$

By Lemma 2.2, we have

$$
\left\|e^{n}\right\|_{\infty} \leq O\left(\tau^{2}+h^{2}\right) .
$$

Similarly to Theorem 4.1 , we can prove the result as follows.

Theorem 4.2. Under the conditions of Theorem 4.1, the solution $u^{n}$ and $\rho^{n}$ of (2.2)-(2.5) is stable in the senses of norm $\|\cdot\|_{\infty}$ and $\|\cdot\|_{L^{2}}$, respectively.

\section{Numerical Simulations}

Since the three-implicit finite difference scheme can not start by itself, we need to select other two-level schemes (such as the C-N Scheme) to get $u^{1}, \rho^{1}$. Then, reusing initial value $u^{0}$, $\rho^{0}$, we can work out $u^{2}, \rho^{2}, u^{3}, \rho^{3}, \ldots$ Iterative numerical calculation is not required, for this scheme is linear, so it saves computing time.

When $t=0$, the damping does not have an effect and the dissipative will not appear. So the initial conditions of (1.4)-(1.7) are same as those of (1.1):

$$
u_{0}(x)=\frac{5}{2} \sec ^{2} \frac{\sqrt{5}}{6} x, \quad \rho_{0}(x)=\frac{5}{3} \sec ^{2} \frac{\sqrt{5}}{6} x, \quad(v=1.5) .
$$


Table 1: The error ratios in the sense of $l_{\infty}$ at various time steps.

\begin{tabular}{ccccc}
\hline & & $\tau=h=0.1$ & $\tau=h=0.05$ & $\tau=h=0.025$ \\
\hline \multirow{4}{*}{$t=0.2$} & $5.783531 e-4$ & $1.366490 e-4$ & $3.178799 e-5$ \\
& $t=0.4$ & $9.505742 e-4$ & $2.237941 e-4$ & $5.198658 e-5$ \\
& $t=0.6$ & $1.159542 e-3$ & $2.724234 e-4$ & $6.320922 e-5$ \\
& $t=0.8$ & $1.246682 e-3$ & $2.925785 e-4$ & $6.789465 e-5$ \\
& $t=1.0$ & $1.248960 e-3$ & $2.936257 e-4$ & $6.817804 e-5$ \\
& $t=0.2$ & $1.292902 e-3$ & $3.176066 e-4$ & $7.553391 e-5$ \\
& $t=0.4$ & $2.182523 e-3$ & $5.367686 e-4$ & $1.277456 e-4$ \\
& $t=0.6$ & $2.182523 e-3$ & $6.760967 e-4$ & $1.610159 e-4$ \\
& $t=0.8$ & $3.046673 e-3$ & $7.521463 e-4$ & $1.792741 e-4$ \\
& $t=1.0$ & $3.154536 e-3$ & $7.796078 e-4$ & $1.859421 e-4$ \\
\hline
\end{tabular}

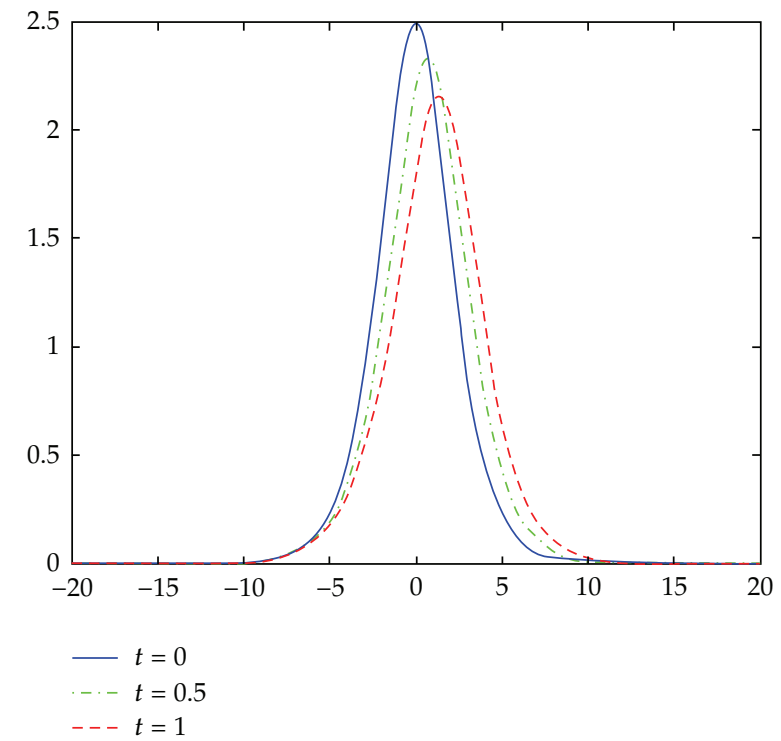

Figure 1: When $\tau=h=0.05$, the wave graph of $u$ at various times.

Let $x_{L}=-20, x_{R}=20, T=1.0$, and $v=\gamma=1$. Since we do not know the exact solution of (1.4)-(1.5), an error estimates method in [21] is used: a comparison between the numerical solutions on a coarse mesh and those on a refine mesh is made. We consider the solution on mesh $\tau=h=1 / 160$ as the reference solution. In Table 1 , we give the ratios in the sense of $l_{\infty}$ at various time steps.

When $\tau=h=0.05$, a wave figure comparison of $u$ and $\rho$ at various time steps is as in Figures 1 and 2.

From Table 1, it is easy to find that the difference scheme in this paper is second-order convergent. Figures 1 and 2 show that the height of wave crest is more and more low with time elapsing due to the effect of damping and dissipativeness. It simulates that the continue energy $E(t)$ of problem (1.4)-(1.7) in Lemma 1.1 is digressive. Numerical experiments show that the finite difference scheme is efficient. 


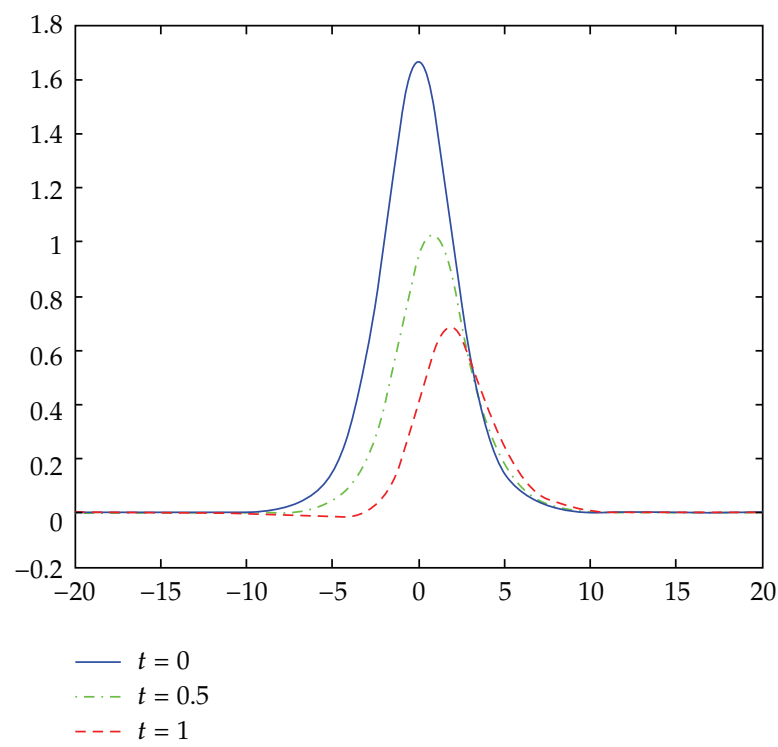

Figure 2: When $\tau=h=0.05$, the wave graph of $\rho$ at various times.

\section{Acknowledgments}

The work of Jinsong Hu was supported by the research fund of key disciplinary of application mathematics of Xihua University (Grant no. XZD0910-09-1). The work of Youcai Xu was supported by the Youth Research Foundation of Sichuan University (no. 2009SCU11113).

\section{References}

[1] C. E. Seyler and D. L. Fenstermacher, "A symmetric regularized-long-wave equation," Physics of Fluids, vol. 27, no. 1, pp. 4-7, 1984.

[2] J. Albert, "On the decay of solutions of the generalized Benjamin-Bona-Mahony equations," Journal of Mathematical Analysis and Applications, vol. 141, no. 2, pp. 527-537, 1989.

[3] C. J. Amick, J. L. Bona, and M. E. Schonbek, "Decay of solutions of some nonlinear wave equations," Journal of Differential Equations, vol. 81, no. 1, pp. 1-49, 1989.

[4] T. Ogino and S. Takeda, "Computer simulation and analysis for the spherical and cylindrical ionacoustic solitons," Journal of the Physical Society of Japan, vol. 41, no. 1, pp. 257-264, 1976.

[5] V. G. Makhankov, "Dynamics of classical solitons (in non-integrable systems)," Physics Reports. Section C, vol. 35, no. 1, pp. 1-128, 1978.

[6] P. A. Clarkson, "New similarity reductions and Painlevé analysis for the symmetric regularised long wave and modified Benjamin-Bona-Mahoney equations," Journal of Physics A, vol. 22, no. 18, pp. 38213848, 1989.

[7] I. L. Bogolubsky, "Some examples of inelastic soliton interaction," Computer Physics Communications, vol. 13, no. 3, pp. 149-155, 1977.

[8] B. Guo, "The spectral method for symmetric regularized wave equations," Journal of Computational Mathematics, vol. 5, no. 4, pp. 297-306, 1987.

[9] J. D. Zheng, R. F. Zhang, and B. Y. Guo, "The Fourier pseudo-spectral method for the SRLW equation," Applied Mathematics and Mechanics, vol. 10, no. 9, pp. 801-810, 1989.

[10] J. D. Zheng, "Pseudospectral collocation methods for the generalized SRLW equations," Mathematica Numerica Sinica, vol. 11, no. 1, pp. 64-72, 1989. 
[11] Y. D. Shang and B. Guo, "Legendre and Chebyshev pseudospectral methods for the generalized symmetric regularized long wave equations," Acta Mathematicae Applicatae Sinica, vol. 26, no. 4, pp. 590-604, 2003.

[12] Y. Bai and L. M. Zhang, "A conservative finite difference scheme for symmetric regularized long wave equations," Acta Mathematicae Applicatae Sinica, vol. 30, no. 2, pp. 248-255, 2007.

[13] T. Wang, L. Zhang, and F. Chen, "Conservative schemes for the symmetric regularized long wave equations," Applied Mathematics and Computation, vol. 190, no. 2, pp. 1063-1080, 2007.

[14] T. C. Wang and L. M. Zhang, "Pseudo-compact conservative finite difference approximate solution for the symmetric regularized long wave equation," Acta Mathematica Scientia. Series A, vol. 26, no. 7, pp. 1039-1046, 2006.

[15] T. C. Wang, L. M. Zhang, and F. Q. Chen, "Pseudo-compact conservative finite difference approximate solutions for symmetric regularized-long-wave equations," Chinese Journal of Engineering Mathematics, vol. 25, no. 1, pp. 169-172, 2008.

[16] Y. Shang, B. Guo, and S. Fang, "Long time behavior of the dissipative generalized symmetric regularized long wave equations," Journal of Partial Differential Equations, vol. 15, no. 1, pp. 35-45, 2002.

[17] Y. D. Shang and B. Guo, "Global attractors for a periodic initial value problem for dissipative generalized symmetric regularized long wave equations," Acta Mathematica Scientia. Series A, vol. 23, no. 6, pp. 745-757, 2003.

[18] B. Guo and Y. Shang, "Approximate inertial manifolds to the generalized symmetric regularized long wave equations with damping term," Acta Mathematicae Applicatae Sinica, vol. 19, no. 2, pp. 191-204, 2003.

[19] Y. Shang and B. Guo, "Exponential attractor for the generalized symmetric regularized long wave equation with damping term," Applied Mathematics and Mechanics, vol. 26, no. 3, pp. 259-266, 2005.

[20] F. Shaomei, G. Boling, and Q. Hua, "The existence of global attractors for a system of multidimensional symmetric regularized wave equations," Communications in Nonlinear Science and Numerical Simulation, vol. 14, no. 1, pp. 61-68, 2009.

[21] B. Hu, Y. Xu, and J. Hu, "Crank-Nicolson finite difference scheme for the Rosenau-Burgers equation," Applied Mathematics and Computation, vol. 204, no. 1, pp. 311-316, 2008. 